\title{
Capsular dissection technique for reducing recurrent laryngeal neuropraxia and temporary hypoparathyroidism after thyroidectomy: a single center experience
}

\author{
Meer M. Chisthi*, Krishnakumar G. Kuttanchettiyar
}

Department of General Surgery, Government Medical College, Trivandrum, Kerala, India

Received: 19 May 2015

Accepted: 24 June 2015

\section{*Correspondence:}

Dr. Meer M. Chisthi,

E-mail: meerchisthi@gmail.com

Copyright: (C) the author(s), publisher and licensee Medip Academy. This is an open-access article distributed under the terms of the Creative Commons Attribution Non-Commercial License, which permits unrestricted non-commercial use, distribution, and reproduction in any medium, provided the original work is properly cited.

\section{ABSTRACT}

Background: Recurrent laryngeal nerve injury and hypoparathyroidism are two of the much discussed major complications of thyroid surgery. It has been classically described by the majority of textbooks to meticulously dissect out the course of the recurrent laryngeal nerve to avoid its injury. However, the argument remains that this dissection might devascularise the nerve and the parathyroids, leading to recurrent laryngeal neuropraxia and temporary hypocalcemia. Hence some authors have recommended adopting a capsular dissection technique in order to avoid these complications.

Methods: This retrospective descriptive study analysed the outcome in a large series of patients who underwent total thyroidectomy by capsular dissection technique, over a 5 year period in a tertiary care center.

Results: As per this study, the incidence of permanent nerve damage was zero whereas the incidence of temporary neuropraxia was found to be around $0.5 \%$. The incidence of temporary and permanent hypocalcemia was found to be around $0.7 \%$ and $0.2 \%$ respectively. These complications were found more in obese patients and those with malignancies.

Conclusions: Taking account of the very low incidence of nerve and parathyroid injury in this series, the authors suggest that meticulous capsular dissection is superior to dissection of the entire nerve in avoiding transcient nerve damage as well as temporary hypocalcemia. However it is stated that a deliberate search for the nerve is definitely indicated in cases where there is likely to be distorted anatomy, as in infiltrating malignancies and recurrent thyroidectomies.

Keywords: Hypocalcemia, Parathyroid, Recurrent laryngeal nerve, Thyroidectomy

\section{INTRODUCTION}

Thyroidectomy is a relatively common major surgical procedure all over the world. The proximity of the gland to the recurrent laryngeal nerve and the parathyroids makes it a procedure with potential complications. Unilateral nerve injury produces hoarseness of voice while bilateral injury is a serious problem and may even cause life threatening stridor. Before the late $20^{\text {th }}$ century, the incidence of recurrent laryngeal nerve injury and permanent hypoparathyroidism were very high after thyroidectomy because the nerve was inadvertently injured during glandular mobilisation. Hence there were a large number of studies which proved that routine nerve dissection and demonstration of the nerve was able to prevent nerve injury. This change in approach has definitely brought down the incidence of permanent injury to the nerve and parathyroids. 
However, recently, many surgeons have noted that routine dissection and visualisation of the nerve produces a high incidence of postoperative temporary nerve damage and also temporary hypocalcemia. Nerve injury may be caused by the injury to the vasa nervosa caused by dissection around the nerve surface. Also, the dissection of the nerve sometimes leads to bleeding which may necessitate ligation of the stem of the inferior thyroid artery to curb bleeding, leading to a higher incidence of parathyroid devascularisation and subsequent hypocalcemia. Usually this hypoparathyroidism resolves over a period of few months but can persist as permanent hypoparathyroidism also, making the patient dependent on life-long calcium supplementation.

The earlier technique of subtotal thyroidectomy was associated with lesser incidence of nerve injury as compared to total thyroidectomy but the higher rates of recurrence has made it unfavourable with surgeons. As per literature review, the incidence of recurrent laryngeal nerve injury has been reported to be around 1 to $2 \%$ when done at thyroid centers by experienced surgeons. However the same complication rate increases to around 6 to $9 \%$ when done by less experienced surgeons. Of this, the rate of permanent nerve injury is around $0.5 \%$ in tertiary care endocrine centers. ${ }^{1,2}$ Surgeon's experience, histopathologic diagnosis, previous thyroid surgery, surgical technique and anatomic variations of the nerve itself have been pointed out as the most important factors affecting this complication. As per literature, the greatest risk factors for permanent nerve palsy are found to be recurrent benign and malignant goiters, technique of no nerve identification, low volume hospital and low volume (inexperienced) surgeons. ${ }^{3}$ Mechanisms of injury to the nerve postulated are complete or partial transection, traction, handling of the nerve, contusion, crushing, electro-cautery burns, clamping, misplaced ligature and compromised blood supply. ${ }^{4}$ Accidental injury to the recurrent laryngeal nerve is found to occur commonly at the level of upper two tracheal rings, where the nerve is closely related to the thyroid lobes in the area of Berry's ligament. ${ }^{5}$ Adequate knowledge of the nerve anatomy and ensuring meticulous hemostasis have been reported to minimise the incidence of nerve injury.

Complete dissection of the recurrent nerve over its entire cervical course has been propounded by many studies on the argument that it allows the surgeon to verify the anatomic integrity of the nerve and to identify extralaryngeal ramifications. However there is conflicting data on whether elaborate nerve dissection is truly beneficial in reducing nerve injury. In fact many studies with nerve identification have given around $1 \%$ incidence of temporary dysphonia. ${ }^{6,7}$ A large volume study of 500 odd patients with deliberate nerve identification reported a temporary palsy of $5 \%$ while that of permanent palsy was $0.9 \% .^{8}$ A study of 350 odd patients sites hematoma and neuropraxia caused by dissection to identify the nerve as the reasons for the 6 cases of temporary nerve injury in their study. In the same study, the surgeons abandoned all attempts to identify the nerve and opted for a capsular dissection when the anatomy was difficult to comprehend, and this sub-group of patients had no postoperative nerve injury. ${ }^{9}$ In fact another study claims that more laryngeal complications are caused by endotracheal intubation than by the surgical technique alone. $^{10}$

In unilateral nerve injury the voice becomes husky because of the vocal cords not approximating with one another. Hoarseness relieved within a few weeks postoperatively is commonly due to edema, whereas traction injury of the nerve and damage of axons may cause dysphonia lasting up to 6 months. Hoarseness persisting after 6 months is usually permanent, caused by dividing, ligating or cauterizing the nerve. ${ }^{11}$ Bilateral nerve injury is much more serious, because both vocal cords may assume a median or para-median position and cause fatal airway obstruction. Such patients will need emergency endotracheal intubation or tracheostomy as the case may be. Though there are lateralization procedures available for the cords, many patients may need a life-long tracheostomy or may become voice-less permanently.

Intra-operative monitoring of the recurrent laryngeal nerve has been touted to be major step in preventing nerve injury by many studies. Combination of electrical RLN stimulation with fibre-optic video laryngoscopy by way of laryngeal mask airway is one of the methods employed to ensure better safety to the nerve. ${ }^{12}$ Some studies have also recommended intra-operative laryngeal palpation as an adjunct to nerve monitoring. ${ }^{13}$ One advantage of RLN monitoring is that neuropraxic injury from extended stretching may be reduced by early warning signals. ${ }^{14}$ In a study directly comparing nerve monitoring with visualisation, monitoring decreased the incidence of transient but not permanent RLN paresis. ${ }^{15}$ Though nerve monitoring is being increasingly used because of the medico-legal implications, it is time consuming as well as expensive. In a large study involving more than 27000 nerves at risk, it was found that the most significant factor in defining outcome was the individual performance of surgeon. ${ }^{16}$ Hence most investigators have recommended neuro-monitoring only for difficult thyroidectomies or repeat operations. ${ }^{17}$

In large volume thyroidectomies, the rates of temporary and permanent hypoparathyroidism rates were found to be around $20 \%$ and $4 \%$ respectively. ${ }^{2}$ With routine exposure of the nerve, the rates have been shown to be $9.5 \%$ and $1.7 \%$ for temporary and permanent hypocalcemia. ${ }^{18}$ A study of around 800 patients cites rates as high as $23 \%$ and $1.6 \%$, implying that routine nerve dissection does compromise the parathyroid blood supply. ${ }^{19}$ This might be explained by the fact that the parathyroids derive their blood supply solely from the inferior thyroid artery. A nerve dissection, when initiating significant bleeding, may lead to hasty efforts to arrest the bleeding, in the form of electro-cauterisation or 
ligation of the artery. Avoidance of nerve dissection might just preserve the precarious blood supply of the parathyroids and avert hypocalcemia.

Capsular dissection, sometimes referred to as Delbridge technique, involves hugging the gland and dividing the tertiary branches of the vessels, while dissecting the parathyroid glands with their vascular pedicles free from the thyroid surface, with minimal exposure of the recurrent laryngeal nerve or disturbance of its blood supply. ${ }^{20}$ When this technique is employed, the nerve is most often encountered within its sheath amongst the tracheo-esophageal groove. In such situations, the surgeon never tries to expose the nerve or dissect in its vicinity. Capsular dissection has been advocated by most authors on the argument that the nerve is not a truly intraglandular structure like the facial nerve and doesn't enter the capsule of the gland. It is separate from the surrounding tissue except following surgical fibrosis or extensive infiltration by malignancy. Hence there is enough surgical sense in following this approach in thyroidectomy.

\section{METHODS}

The objective of the study was to analyse the incidence of temporary as well as complications related to the recurrent laryngeal nerve and the parathyroids, in patients undergoing total thyroidectomy by Capsular dissection technique. This study is being reported from the General Surgery unit of Government Medical college, Trivandrum, a tertiary care teaching hospital. Being one of the largest health care institutions in the state, this hospital caters to a large number of patients every year. This is a Retrospective descriptive study. The authors, both of whom are general surgeons, have been independently doing thyroidectomies for over 5 years. From January 2009 to December 2014, over a period of 5 years, all patients operated for thyroid disease by both the surgeons were included for the observation. The inclusions were all total thyroidectomy patients, aged 2080 years, who were operated by Capsular dissection by the surgeons over the study period. Any patient with previous recurrent laryngeal nerve pathology or parathyroid disease were excluded. Data regarding the patients was collected by analysing the case sheets available at the Medical records library of the hospital.

All the patients had a preoperative vocal cord assessment by indirect laryngoscopy. The patients were informed about the potential complications and informed written consent obtained before surgery. The surgical technique was noted as follows. Kocher's collar incision was employed to incise the skin. Once the flaps were raised, the deep fascia was opened. After ligation of middle thyroid vein and the superior pedicle, the capsular dissection was commenced. The thyroid lobe was dissected in close proximity to the capsule. The branches of inferior thyroid artery were ligated inside the capsule, thus preserving the parathyroid vascularity. After preserving the parathyroids along with its blood supply, the thyroid lobe was dissected off the trachea by sharp dissection. Bleeding points were either carefully ligated or cauterised by bipolar diathermy. No deliberate attempt was made to dissect out the recurrent laryngeal nerve. However, in most cases, it was seen traversing the tracheoesophageal groove, within its membranous fascia, entering the larynx close to the Berry's ligament. The fascia was left intact to prevent nuropraxia. Once the lobar dissection was completed, the inferior pole was ligated and the gland delivered out. After ensuring hemostasis, the wound was closed, with a drain in most cases. In all patients, the vocal cords were assessed by direct laryngoscopy by the anesthetist before extubation. The drain was removed on the second or third postoperative day and sutures removed on fifth day.

Postoperatively, the patients were monitored for any voice change for a period of 1 year. Patients who reported hoarseness were subjected to indirect laryngoscopy to assess the vocal cords. Vocal cord immobility was deemed as recurrent laryngeal nerve palsy and they were advised voice rest and other supportive measures. In patients with voice complaints, follow - up laryngoscopy was done at 3, 6 and 12 months to assess cord function. Serum calcium levels were done on every day till the fifth postoperative day in all patients. Also, the serum calcium was estimated whenever the patient reported symptoms of hypocalcemia like numbness of hands and feet. Documented hypocalcemia patients received oral calcium supplementation. Temporary hypoparathyroidism was diagnosed when the patients could be weaned off calcium supplementation within 6 months after surgery.

The resultant data was entered into a prefixed proforma. All statistical analyses were performed using SPSS (SPSS Inc., USA). The $\chi^{2}$ test or Fisher exact test and independent 2-tailed t-tests were used to compare the clinico-pathological parameters and surgical outcomes, wherever appropriate. Continuous variables were stratified and analyzed as categorical data. The level of statistical significance was set at $\mathrm{p}<0.05$.

\section{RESULTS}

In this study, females outnumbered males by a ratio of 23.4. This is explained by the fact that a majority of the patients who develop goiters as well as those who demand surgery for cosmetic reasons are females. Most of the patients belonged to the 40 - 60 age group, followed by the 20-40 group (Figure 1). The mean value of age was 42.7. The mean weight was $61.7 \mathrm{~kg} .37$ patients had a BMI more than 30 .

The maximum number of patients had a preoperative diagnosis of colloid goiter, followed by lymphocytic thyroiditis. The least number of patients had malignancy as their preoperative diagnosis (Figure 2). There were only two cases of revision thyroidectomies implying that 
the surgeons opted out of capsular dissection and went for nerve demonstration in such cases.

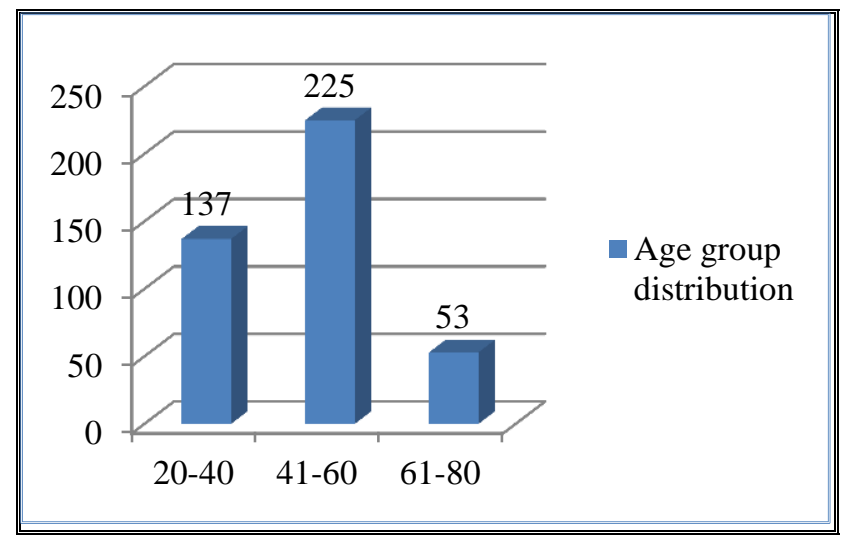

Figure 1: Age group distribution.

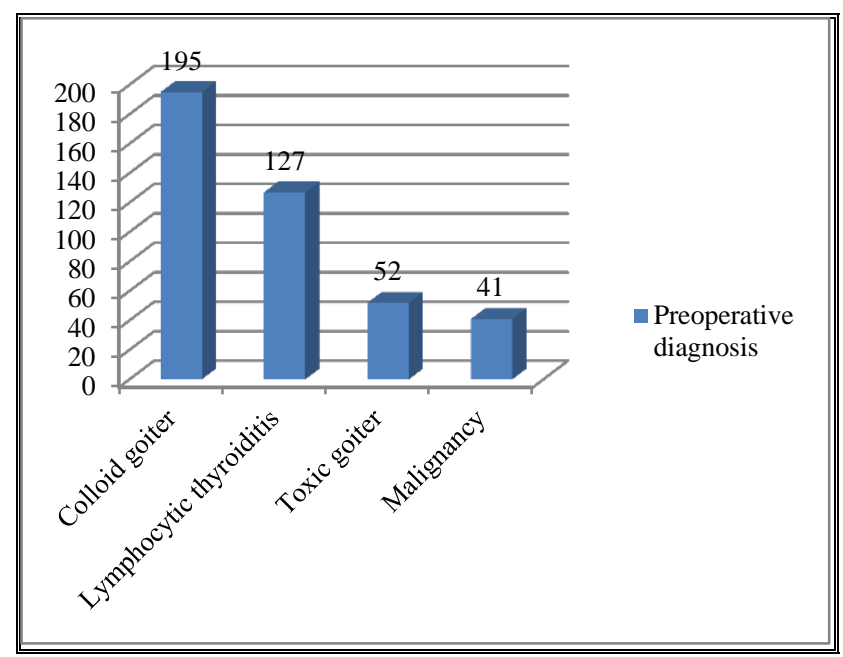

Figure 2: Preoperative diagnosis.

In the series, there were no cases of permanent recurrent laryngeal nerve palsy. Both surgeons had 1 case of unilateral temporary nerve palsy each, which resolved spontaneously over a period of few weeks $(0.48 \%)$. These patients had lymphocytic thyroiditis or malignancy as their preoperative diagnosis. Both these patients improved over the period of a few weeks and none of the palsies persisted beyond 6 months. Of the 415 patients, there were 3 cases of temporary hypoparathyroidism $(0.72 \%)$ and only 1 case of permanent hypoparathyroidism (0.24\%) (Figure 3). All of these patients received calcium supplementation and improved symptomatically. The 1 patient with permanent hypoparathyroidism was put on long-term calcium suplementation.

The other noted complications included upper respiratory infection $(5.78 \%)$, flap hematoma $(2.41 \%)$, wound infection (1.20\%) and lower respiratory infection $(0.72 \%)$. 1 patient developed acute cerebrovascular accident during the second postoperative day (Figure 4). There were no deaths within the follow-up period.

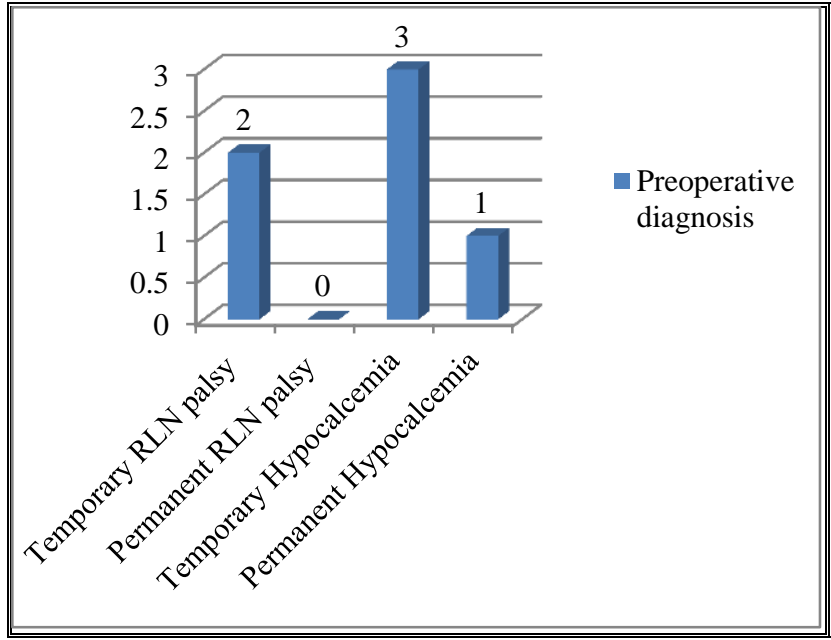

Figure 3: Recurrent laryngeal nerve and Parathyroid complications.

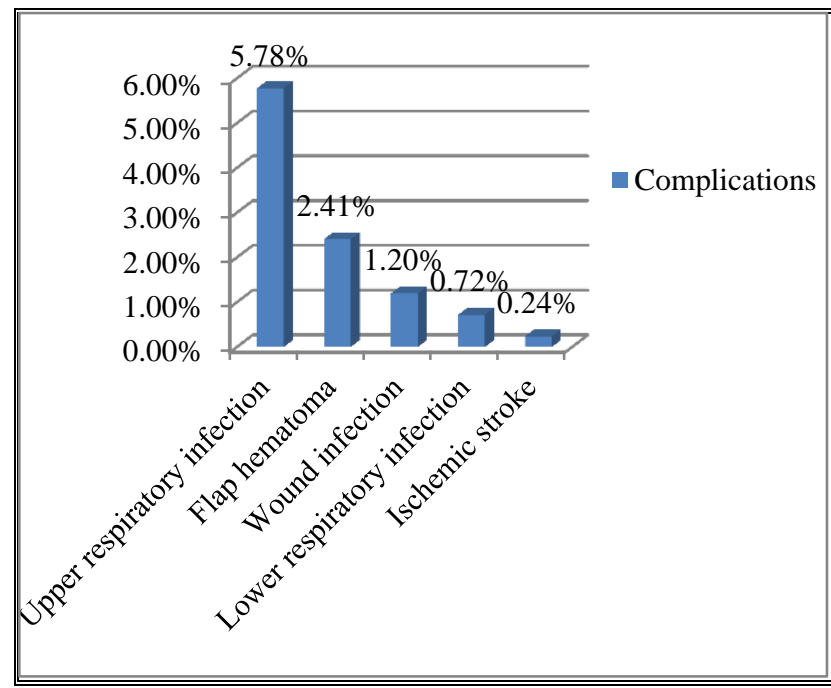

Figure 4: Other complications.

Of the 415 patients, 387 were available for follow - up for 1 year postoperatively. 14 patients discontinued followup after 6 months whereas 14 patients dropped out after 9 months. However all those patients who had any form of nerve injury or hypocalcemia remained on follow-up till the end of 1 year period.

The patient characteristics associated with temporary nerve injury included an age group within 41-60 years and a preoperative diagnosis of lymphocytic thyroiditis or malignancy. The characteristics associated with both temporary and permanent parathyroid insufficiency included age group within 61-80 years, obesity and a preoperative diagnosis of lymphocytic thyroiditis or malignancy (Table 1). On further analysis, no factor was found to be statistically significant with regard to temporary nerve injury. However, an elderly age, obesity and a diagnosis of malignancy were found to be of statistical significance in causing hypoparathyroidism. 
Table 1: Patient characteristics associated with RLN and PT complications.

\begin{tabular}{|llllllll|} 
& Number & $\begin{array}{l}\text { RLN } \\
\text { palsy }\end{array}$ & $\begin{array}{l}\text { Odds } \\
\text { Ratio }\end{array}$ & P value & $\begin{array}{l}\text { PT } \\
\text { insufficiency }\end{array}$ & $\begin{array}{l}\text { Odds } \\
\text { Ratio }\end{array}$ & P value \\
\hline Age 41-60 & 225 & 2 & 4.26 & 0.35 & 1 & 0.42 & 0.48 \\
\hline Age 61-80 & 53 & 0 & 1.35 & 0.85 & 2 & 14.16 & 0.03 \\
\hline Obesity & 37 & 1 & 10.47 & 0.10 & 3 & 76.80 & 0.004 \\
\hline $\begin{array}{l}\text { Lymphocytic } \\
\text { thyroiditis }\end{array}$ & 127 & 1 & 2.28 & 0.56 & 1 & 1.13 & 0.92 \\
\hline Malignancy & 41 & 1 & 9.32 & 0.12 & 2 & 19.13 & 0.02 \\
\hline
\end{tabular}

\section{DISCUSSION}

As per the results of this study, the incidence of temporary as well as permanent recurrent laryngeal nerve injury and hypoparathyroidism are found to be less than that of other reported studies. Also, it was noted that those patients who had temporary nerve palsy and hypoparathyroidism had either lymphocytic thyroiditis or malignancy or were obese. This observation might be explained by the fact that both malignancy and lymphocytic thyroiditis might distort the normal anatomy and cause difficulties in ensuring a proper safeguarding of the nerve and the parathyroids. The association with obesity might be because of the inadequate neck extension and subsequent visibility that can be achieved while positioning these patients for the surgery.

Hence we would like to reaffirm the argument that a capsular dissection technique might avoid neuropraxia and temporary nerve damage in routine thyroidectomies. However this study has a drawback in that the surgeries were conducted by two experienced thyroid surgeons and hence may not be reproducible ubiquitously. Also, we do admit that a large volume multi-centric trial might be necessary to validate our conclusions before it can be applied generally. If such studies are undertaken and proven correct, large number of patients can be spared of the distressing after effects of temporary nerve damage.

In conclusion, the authors state that temporary nerve injury by capsular dissection technique was much less than that quoted in the literature. The rate of hypoparathyroidism was also proportionately low. The conventional technique of nerve dissection might be reserved for recurrent surgeries and infiltrating neoplasms as the anatomy might be distorted in such cases. However we reiterate the fact that sound knowledge of anatomy and ensuring adequate hemostasis are of paramount importance in preventing complications during thyroidectomy. The authors feel that this study might initiate a shift in paradigm in thyroid surgery.

\section{ACKNOWLEDGMENTS}

The authors would like to thank the General Surgery department of Government Medical college, Trivandrum and all the faculty, especially the Head of the department Prof. Sreekumar A, for all the support in conducting this study.

Funding: No funding sources

Conflict of interest: None declared

Ethical approval: Not required

\section{REFERENCES}

1. Delbridge L, Guinea AI, Reeves TS. Total thyroidectomy for benign multinodular goiter. Arch Surg. 1999; 134:1389-93.

2. Bergamaschi R, Becouarn G, Ronceray J, Arnaud JP. Morbidity of thyroid surgery. Am J Surg. 1998 July;176(1):71-5.

3. Dralle H, Sekulla C, Haerting J, Timmermann W, Neumann HJ, Kruse E, et al. Risk factors of paralysis and functional outcome after recurrent laryngeal nerve monitoring in thyroid surgery. Surgery. 2004 Dec;136(6):1310-22.

4. Rice DH, Cone-Wesson B. Intraoperative recurrent laryngeal nerve monitoring. Otolaryngol Head Neck Surg. 1991 Sep;105(3):372-5.

5. Marcus B, Edwards B, Yoo S, Byrne A, Gupta A, Kandrevas $\mathrm{J}$, et al. Recurrent laryngeal nerve monitoring in thyroid and parathyroid surgery: the University of Michigan experience. Laryngoscope. 2003 Feb;113(2):356-61.

6. Sturniolo G, D'Alia C, Tonante A, Gagliano E, Taranto F, Schiavo MGL. The recurrent laryngeal nerve related to thyroid surgery. Am J Surg. 1999 Jun;177(6):485-8.

7. Shindo ML, Wu JC, Park EE. Surgical anatomy of the recurrent laryngeal nerve revisited. Otolaryngol Head Neck Surg. 2005 Oct;133(4):514-9.

8. Chiang FY, Wang LF, Huang YF, Lee KW, Kuo WR. Recurrent laryngeal nerve palsy after thyroidectomy with routine identification of the recurrent laryngeal nerve. Surgery. 2005 Mar;137(3):342-7.

9. Nour AM, Al-Momen AHA. Prevention of recurrent laryngeal nerve injury in thyroid surgery: are neuromonitoring techniques needed? East Central Afr J Surg. 2011 Mar/Apr;16(1):1-9.

10. Echternach M, Maurer CA, Mencke T, Schilling M, Verse T, Richter B. Laryngeal complications after 
thyroidectomy: is it always the surgeon? Arch Surg. 2009 Feb;144(2):149-53.

11. Bailleux S, Bozec A, Castillo L, Santini J. Thyroid surgery and recurrent laryngeal nerve monitoring. J Laryngol Otol. 2006 Jul;120(7):566-9.

12. Shindo ML. Considerations in surgery of the thyroid gland. Otolaryngol Clin North Am. 1996;29:629-35.

13. Randolph GW, Kobler JB, Wilkins J. Recurrent laryngeal nerve identification and assessment during thyroid surgery: laryngeal palpation. World J Surg. 2004 Aug;28(8):755-60.

14. Shindo M, Chheda NN. Incidence of vocal cord paralysis with and without recurrent laryngeal nerve monitoring during thyroidectomy. Arch Otolaryngol Head Neck Surg. 2007;133:481-5.

15. Barczyński M, Konturek A, Cichoń S. Randomized clinical trial of visualization versus neuromonitoring of recurrent laryngeal nerves during thyroidectomy. Br J Surg. 2009 Mar;96(3):240-6.

16. Hermann M, Alk G, Roka R, Glaser K, Freissmuth M. Laryngeal recurrent nerve injury in surgery for benign thyroid diseases. Ann Surg. 2002 Feb;235(2):261-8.

17. Beldi G, Kinsbergen T, Schlumph R. Evaluation of intraoperative recurrent nerve monitoring in thyroid surgery. World J Surg. 2004;28:589-91.

18. Al-Fakhri N, Schwartz A, Runkel N, Buhr HJ. Rate of complications with systematic exposure of the recurrent laryngeal nerve and parathyroid glands in operations for benign thyroid gland diseases. Zentralbl Chir. 1998;123(1):21-4.

19. Nair CG, Babu MJ, Menon R, Jacob P. Hypocalcaemia following total thyroidectomy: an analysis of 806 patients. Indian J Endocr Metab. 2013;17:298-303.

20. Delbridge L, Reeve TS, Khadra M, Poole AG. Total thyoidectomy: the technique of capsular dissection. Austr N Z J Surg. 1992 Feb;62(2):96-9.

Cite this article as: Chisthi MM, Kuttanchettiyar KG. Capsular dissection technique for reducing recurrent laryngeal neuropraxia and temporary hypoparathyroidism after thyroidectomy: a single center experience. Int J Otorhinolaryngol Head Neck Surg 2015;1:17-22. 